\title{
MRI Study of Cerebral Cortical Thickness in Patients with Herpes Zoster and Postherpetic Neuralgia
}

\author{
Xian Liu $\mathbb{D}^{\prime}{ }^{\prime *}$, Lili Gu², Jiaqi Liu³, Shunda Hong', Qing Luo', Ying Wu (D)', Jiaojiao Yang', Jian Jiang' \\ 'Department of Radiology, The First Affiliated Hospital, Nanchang University, Nanchang, Jiangxi, People's Republic of China; ${ }^{2}$ Department of Pain, The \\ First Affiliated Hospital, Nanchang University, Nanchang, Jiangxi, People's Republic of China; ${ }^{3}$ Department of Radiology, The Jiangxi Provincial People's \\ Hospital, Nanchang, Jiangxi, 330006, People's Republic of China
}

*These authors contributed equally to this work

Correspondence: Jian Jiang, Department of Radiology, The First Affiliated Hospital, Nanchang University, 17 Yongwaizheng Street, Nanchang, 330006 , Jiangxi, People's Republic of China, Tel +86 79 I 8869 3825, Email jij2002cn@I26.com

Objective: To measure the changes in cerebral cortical thickness in patients with herpes zoster (HZ) and postherpetic neuralgia (PHN) by surface-based morphometry (SBM) and further estimate its correlation with clinical scores.

Materials and Methods: Twenty-nine HZ patients, $30 \mathrm{PHN}$ patients and 30 well-matched healthy controls (HCs) were included. Magnetic resonance imaging (MRI) data from all subjects were collected and then analyzed by SBM. The changes in cortical thickness among the HZ, PHN and HC groups were analyzed by ANOVA and correlated with clinical scores.

Results: The thickness of the bilateral primary visual cortex (V1, V2) and right primary visual cortex (V3), left somatosensory cortex (L3A), right anterior cingulate gyrus and medial prefrontal cortex (RS32) increased in PHN group, and the thickness the left insular and frontal opercular cortex (LFOP4), left motor cortex (L3B), and right superior temporal visual cortex (RSTV) were decreased in the $\mathrm{HZ}$ and PHN groups compared to the HC group. The thickness measurements of RS32, LFOP4, and (L3B) in HZ and PHN patients were correlated with the duration of disease. In HZ and PHN patients, the Hamilton Anxiety Scale (HAMA) and Hamilton Depression Scale (HAMD) scores were significantly positively correlated.

Conclusion: Changes in cortical thickness in the areas related to sensory, motor, and cognitive/emotional changes in patients with PHN affect the neuroplasticity process of the brain, which may be the reason for the transformation of HZ into PHN and provide a possible explanation for the neuropathological mechanism of pain persistence in PHN patients.

Keywords: postherpetic neuralgia, PHN, neuropathic pain, cortical thickness, surface-based morphometry, SBM

\section{Introduction}

Herpes zoster (HZ) is a disease in which latent varicella zoster virus (VZV) is reactivated in the dorsal root ganglion, resulting in the distribution of herpes on the skin innervated by the relevant neurons. Postherpetic neuralgia (PHN) is the most common complication of herpes zoster. PHN is a typical form of chronic neuropathic pain that is still painful after the disappearance of acute HZ skin lesions and lasts more than 1 month. Its main clinical manifestation is severe pain, often accompanied by anxiety and depression. At present, the mechanism of PHN is not clear, resulting in poor therapeutic effectiveness and repeated attacks of disease. However, studies have shown that neuropathic pain is a direct effect of structural injury or disease of the peripheral or central nervous system desogestrel. ${ }^{1}$ The pathological basis of PHN consists of neuroplastic changes in the peripheral and central nervous system; the most important mechanism is central sensitization. ${ }^{2}$

Owing to the rapid development of neuroimaging, magnetic resonance imaging (MRI) plays an increasingly important role in the study of the central nervous system and neuropathic pain. Its advantage is noninvasive and radiation-free. It has been widely used to study the neural mechanism of various neuropathic pain diseases. At present, MRI research on $\mathrm{HZ}$ is divided into two main research directions: functional research and structural research. There are far fewer studies on structural MRI than functional MRI of the brains of patients with HZ, especially cortex-related 
research. Previous studies have shown that the brain structure is mainly composed of the medulla and cortex. Previous studies on the brain medulla mainly focused on diffusion tensor imaging (DTI), ${ }^{3,4}$ which found that the microstructural integrity of white matter in multiple brain regions of PHN patients changed. Previous studies on the cerebral cortex of herpes zoster mainly included diffusion kurtosis imaging (DKI) and voxel-based morphometry (VBM). A DKI study ${ }^{5}$ found alterations in the pathophysiological microstructure of gray matter in patients with PHN. A VBM study ${ }^{6,7}$ found extensive gray matter volume (GMV) abnormalities in HZ and PHN patients.

There are two main latest research methods on resting-state MRI of cerebral cortex morphology; one is VBM, and the other is surface-based morphometry (SBM). Both have been widely used to study brain morphology under normal and pathological conditions. ${ }^{8-12}$ However, there are few studies on the surface morphology of the HZ and PHN based on the cerebral cortex. Compared with the traditional VBM method, SBM can achieve more accurate spatial positioning than traditional VBM. ${ }^{13}$ Since the VBM study found a wide range of GMV abnormalities in HZ and PHN patients, ${ }^{6,7}$ and the GMV reflects comprehensive information on cortical surface area, cortical folding and cortical thickness, of which the latter is closely related to volume; ${ }^{14}$ we can assume that there may be a wide range of brain areas with cortical thickness changes in HZ and PHN patients.

\section{Materials and Methods Subjects}

The participating patients, comprising 29 with $\mathrm{HZ}$ and 30 with PHN, were recruited from the pain department of the First Affiliated Hospital of Nanchang University. The pain sites of the patients were diverse and were mainly distributed in the bilateral head, face, chest, back and limbs, of which 25 lesions were located on the left side of the body and 34 on the right side of the body. Pain intensity was assessed using a visual analog scale (VAS) before the fMRI scan, ranging from 0 to 10, indicating pain intensity from no pain to the highest intolerable pain. The Hamilton Depression Scale (HAMD) was used to evaluate the degree of depression, and the Hamilton Anxiety Scale (HAMA) was used to evaluate the degree of anxiety. A list of 90 symptoms was used to evaluate psychological or emotional disorders.

The inclusion criteria for HZ and PHN patients were as follows: (1) The patients were 30 85 of years. (2) All patients were right-handed. (3) According to the diagnostic criteria defined by the International Association for Pain Research, the diagnostic criterion for $\mathrm{HZ}$ is that the lesions appear on the skin and do not subside within 2 weeks. The diagnostic criterion for PHN is that the pain lasts for more than 1 month after the recovery of HZ skin lesions; it was diagnosed by two consultant physicians in the pain department. (4) All HZ and PHN patients had a pain score of $\geq 5$. (5) No painkillers were taken at least 2 months before MRI. The exclusion criteria were as follows: (1) other chronic pain diseases; (2) other mental or neurological diseases; (3) a history of head injury, alcoholism or drug abuse; (4) failed scans or poor cooperation during MRI; and (5) claustrophobia. Thirty healthy right-handed controls matched with HZ and PHN patients for age and sex, ranging in age from 56 to 78 years and lacking any spontaneous pain or sensory abnormalities, substance abuse, neurological disorders or significant mental disorders were recruited. Written informed consent was obtained from all participants or their guardians.

This study was reviewed and approved by the medical research ethics committee and institutional review board of the First Affiliated Hospital of Nanchang University and conformed to the principles stipulated in the Declaration of Helsinki.

\section{Image Acquisition}

MRI data were collected on a 3.0 T Siemens TIM Trio scanner (Erlangen, Bavaria, Germany) in the radiology department of the First Affiliated Hospital of Nanchang University. Three-dimensional high-resolution T1-weighted images of the brain were collected at 176 sagittal positions in each participant. The gradient-echo sequence had the following parameters: repetition time $=1900 \mathrm{~ms}$, echo time $=2.26 \mathrm{~ms}$, flip angle $=9^{\circ}$, matrix $=240 \times 256$, field of view $=215 \times 230 \mathrm{~ms}$, slice thickness $=1.0 \mathrm{~mm}$, slice gap $=0 \mathrm{~mm}$, scanning time $=3: 34$ minutes. During the MRI scans, participants were required to close their eyes but remain awake. Earplugs were available to reduce the noise of the machine. At the same time, conventional T2-weighted images were used to exclude visible brain structural abnormalities. 


\section{Cortical Thickness Analysis}

The main method of cortical thickness analysis is SBM, whose research tools currently mainly include FreeSurfer and DPABISurf software.DPABIsurf (version 1.6; http://rfmri.org/dpabi) is a resting-state fMRI data analysis toolbox that calculates cortical thickness from 3D T1-weighted imaging (T1WI). The software is an upgraded version based on fmriprep20.2.1 and FreeSurfer software. It integrates multiple functional interfaces for preprocessing, statistical analysis and result viewing. DPABIsurf is a mature and reliable tool for resting-state functional brain MRI research. In brief, the preprocessing procedure consists of registering the T1WI scans to the Talairach space; performing offset field correction; removing the non-brain structures by skull stripping; segmenting the brain tissue into white matter, gray matter and cerebrospinal fluid; identifying the boundary between gray matter and white matter in each cortical hemisphere; calculating the white matter/gray matter and gray matter/cerebrospinal fluid ratios; expanding and deforming the cortical surface into a sphere; resampling onto the average surface; and smoothing the data with a $10 \mathrm{~mm}$ full width at half maximum Gaussian kernel to reduce measurement error. Cortical thickness was calculated by measuring the distance between the white matter/gray matter boundary and the gray matter/pia mater boundary. The built-in template for the division of cortical anatomical regions in DPABIsurf software is from the Human Connectome Project (HCP).

\section{Statistical Analysis}

Statistical analysis was carried out in SPSS 22 software. The normality of different variables in the three samples was confirmed by the Kolmogorov-Smirnov test, and the homogeneity of variance was evaluated by Levene's test. ANOVA was used to compare the differences in age, sex, VAS scores, 90-symptom list scores, HAMA scores, HAMD scores and cortical thickness. Age and sex were used as covariates when cortical thickness was compared among HZ patients, PHN patients, and healthy controls (HCs) using analysis of variance (ANOVA). After a false detection rate (FDR) correction for multiple comparisons, $\mathrm{P}<0.05$ was considered statistically significant. If no significant clusters survived the correction for multiple comparisons, the uncorrected statistical threshold was also reported as a change trend. Pearson correlation analysis was used to further investigate the relationship between cortical thickness and clinical data in patients with HZ and PHN.

\section{Results}

\section{Demographic and Clinical Characteristics}

We found that there was no significant difference in age $(\mathrm{F}=1.59, \mathrm{P}=0.21)$ or $\operatorname{sex}(\mathrm{F}=1.05, \mathrm{P}=0.35)$ among $\mathrm{HZ}$ patients, PHN patients, and HCs. There was no significant difference in VAS scores ( $\mathrm{F}=1.41, \mathrm{P}=0.24)$, 90-symptom list scores $(\mathrm{F}=2.47, \mathrm{P}=0.12)$ or HAMA scores $(\mathrm{F}=2.71, \mathrm{P}=0.11)$ between $\mathrm{HZ}$ and PHN patients. There were significant differences in disease duration $(\mathrm{F}=4.17, \mathrm{P}=0.046)$ and HAMD scores $(\mathrm{F}=5.694, \mathrm{P}=0.020)$ between $\mathrm{HZ}$ and PHN patients (Table 1).

\section{Cortical Thickness Alterations}

When age and gender were used as covariates, the thickness of the bilateral primary visual cortex (V1 and V2), right primary visual cortex (RV3), left somatosensory and motor cortex (L3A and L3B), and right anterior cingulate and medial prefrontal cortex (RS32) increased in the PHN group compared to the HC group, while the thickness of the left insular and frontal opercular cortex (LFOP4) and right superior temporal visual cortex (RSTV) decreased in PHN group (all corrected by FDR, Table 2, Figures 1 and Figure 2). Compared with the HZ group, PHN patients showed increased cortical thickness in LV1 and L3A, and the cortical thickness of RSTV, LFOP4 and L3B decreased $(\mathrm{P}<0.05$, not corrected by FDR, Table 2 and Figure 2). Compared with the values from the HC group, the cortical thickness of bilateral V1, V2 and left L3A in HZ patients increased, and the cortical thickness of RSTV and L3B decreased $(\mathrm{P}<0.05$, not corrected by FDR, Table 2 and Figure 2). 
Table I Demographic and Clinical Characteristics of the Sample

\begin{tabular}{|l|l|l|l|l|l|}
\hline & HZ (n=29) & PHN (n=30) & HC (n=30) & F & P \\
\hline Age (years) & $62.4 \pm 10.4$ & $66 \pm 10.7$ & $63 \pm 9.0$ & 1.59 & 0.21 \\
Sex (M/F) & $19 / 10$ & $17 / 13$ & $14 / 16$ & 1.05 & 0.35 \\
VAS score & $6.1 \pm 0.9$ & $6.4 \pm 0.9$ & - & 1.41 & 0.24 \\
Duration of disease (months) & $0.35 \pm 0.17$ & $5.43 \pm 13.39$ & - & 4.17 & 0.046 \\
90-symptom list score & $124.6 \pm 18.4$ & $136.7 \pm 37.3$ & - & 2.47 & 0.12 \\
HAMA score & $13.7 \pm 6.4$ & $17.3 \pm 9.7$ & - & 2.71 & 0.11 \\
HAMD score & $14.9 \pm 4.5$ & $19.3 \pm 8.9$ & - & 5.69 & 0.02 \\
\hline
\end{tabular}

Abbreviations: HZ, herpes zoster; PHN, postherpetic neuralgia; HC, healthy control; M/F, male/female; HAMA, Hamilton Anxiety Scale; HAMD, Hamilton Depression Scale; VAS, visual analog scale.

Table 2 Brain Regions with Significant Changes from HC Cortical Thickness in Patients with HZ and PHN

\begin{tabular}{|c|c|c|c|c|c|c|c|c|c|}
\hline \multirow[b]{2}{*}{ Brain Regions } & \multirow[b]{2}{*}{ Right/Left Hemisphere } & \multirow[b]{2}{*}{ Cluster Size (mm) } & \multirow[b]{2}{*}{ MNI Coordinates $(x, y, z)$} & \multicolumn{2}{|c|}{$\mathbf{H Z}$} & \multicolumn{2}{|c|}{ PHN } & \multicolumn{2}{|c|}{$\mathrm{HC}$} \\
\hline & & & & Mean & SD & Mean & SD & Mean & SD \\
\hline RVI & $\mathrm{R}$ & 174.37 & $7.18014,-75.7524,13.5319$ & 1.52 & 0.10 & 1.54 & 0.17 & 1.45 & 0.95 \\
\hline $\mathrm{RV} 2$ & $\mathrm{R}$ & 140.65 & $12.4656,-70.0993,-6.37935$ & 1.71 & 0.14 & 1.75 & 0.13 & 1.60 & 0.16 \\
\hline $\mathrm{RV} 3$ & $\mathrm{R}$ & 57.24 & $9.37741,-75.3772,23.188$ & 1.79 & 0.16 & 1.81 & 0.15 & 1.70 & 0.15 \\
\hline RS32 & $\mathrm{R}$ & 18.35 & $6.6223,30.572,-8.49025$ & 2.60 & 0.29 & 2.76 & 0.48 & 2.47 & 0.24 \\
\hline RSTV & $\mathrm{R}$ & 19.61 & $61.564 \mathrm{I},-36.4483,9.25996$ & 2.58 & 0.39 & 2.36 & 0.34 & 2.86 & 0.33 \\
\hline LVI & $\mathrm{L}$ & 125.11 & $62.0247,-48.9723,-2.08364$ & 1.49 & 0.06 & 1.51 & 0.09 & 1.43 & 0.08 \\
\hline LV2 & L & 68.28 & $47.3374,-60.1029,20.667$ & 1.62 & 0.09 & 1.65 & 0.13 & 1.56 & 0.09 \\
\hline L3A & $\mathrm{L}$ & 18.23 & $|2.508|,-16.9894,67.2258$ & 2.50 & 0.20 & 2.30 & 0.25 & 2.62 & 0.17 \\
\hline L3B & L & 15.05 & $32.3518,-30.4993,58.5086$ & 1.75 & 0.32 & 1.56 & 0.34 & 1.84 & 0.29 \\
\hline LFOP4 & L & 16.48 & $6.85307,29.1373,-15.1879$ & 1.59 & 0.25 & 1.41 & 0.26 & 1.71 & 0.26 \\
\hline
\end{tabular}

Notes: color bar: warm colors indicate increased cortical thickness, and cold colors indicate decreased cortical thickness.

Abbreviations: VI, V2, V3, primary visual cortex; LFOP4, left insular and frontal opercular cortex; RS32, right anterior cingulate gyrus and medial prefrontal cortex; RSTV, right superior temporal visual cortex; L3A, L3B, left somatosensory and motor cortex.

\section{Correlation Analysis}

There was a positive correlation between the thickness of RS32 $(r=0.537, \mathrm{P}<0.001)$ and the duration of disease in $\mathrm{HZ}$ and PHN patients (Figure 3). There was a negative correlation linking the thickness values of the LFOP4 $(\mathrm{r}=-0.28, \mathrm{P}=0.033)$ and left motor cortex $\mathrm{L} 3 \mathrm{~B}(\mathrm{r}=-0.2, \mathrm{P}=0.027)$ to the duration of disease in $\mathrm{HZ}$ and PHN patients (Figure 3). There was a significant correlation between the HAMA and HAMD scores in HZs and PHN patients $(r=0.78, \mathrm{P}<0.001$, Figure 4).

\section{Discussion}

The cerebral cortex is the most important part of the brain, has many functional centers and is the basis of high-level neural activities.In this study, SBM was used to study the changes in the thickness of the cerebral cortex in HZ and PHN patients. Our main finding was that there were significant differences in the thickness of the bilateral V1 and V2, L3A and L3B, RS32, LFOP4, and RSTV in PHN patients compared to HCs, which may be related to pain, anxiety, and depression symptoms in patients.

The insula belongs to the "significance network" and is the key area for response and emotion regulation to significant events such as pain. The insula plays an important role in human sensory and motor function, pain, emotion, cognition and decision-making. ${ }^{15}$ Previous studies have shown that reduced insular cortical thickness plays an important role in patients with pain-related diseases such as somatoform pain disorder (SPD) ${ }^{16}$ and trigeminal neuralgia. ${ }^{17}$ The temporal lobe is considered to play a very important role in mood regulation disorders (anxiety, depression) and language memory. ${ }^{18,19}$ Thinning of the superior temporal gyrus cortex is also found in depression, ${ }^{20}$ trigeminal neuralgia, ${ }^{21}$ and chronic pain disorder (CPD). ${ }^{19}$ The present study also found that the thickness of LFOP4 and RSTV decreased in PHN 


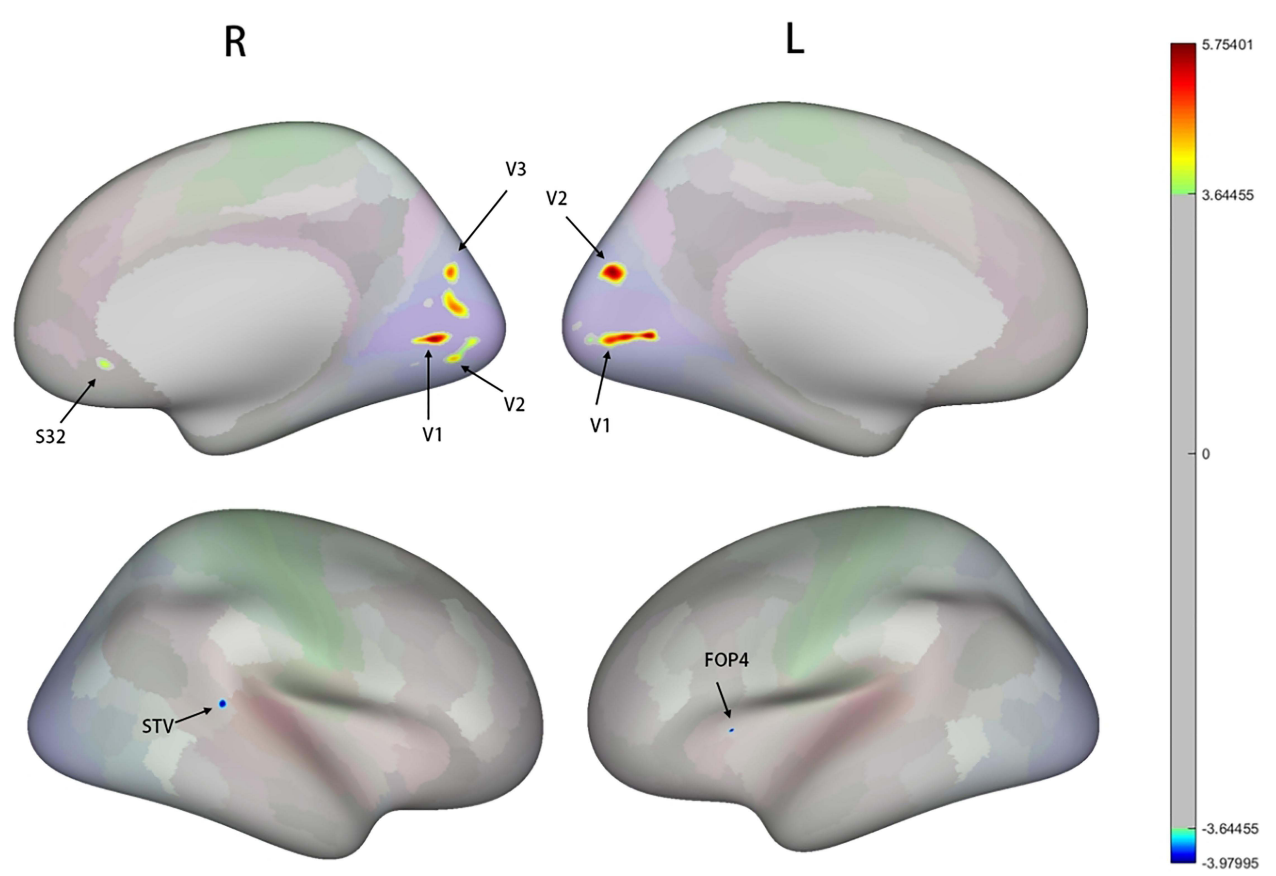

Figure I Brain regions with significant differences in cortical thickness in PHN patients compared with HCs.

Notes: 3a, 3b, somatosensory and motor cortex, not shown in the figure; color bar, warm colors indicate increased cortical thickness, and cold colors indicate decreased cortical thickness.

Abbreviations: R, right hemisphere; L, left hemisphere; VI, V2, V3, primary visual cortex; FOP4, insular and frontal opercular cortex; S32, anterior cingulate gyrus and medial prefrontal cortex; STV, superior temporal visual cortex.

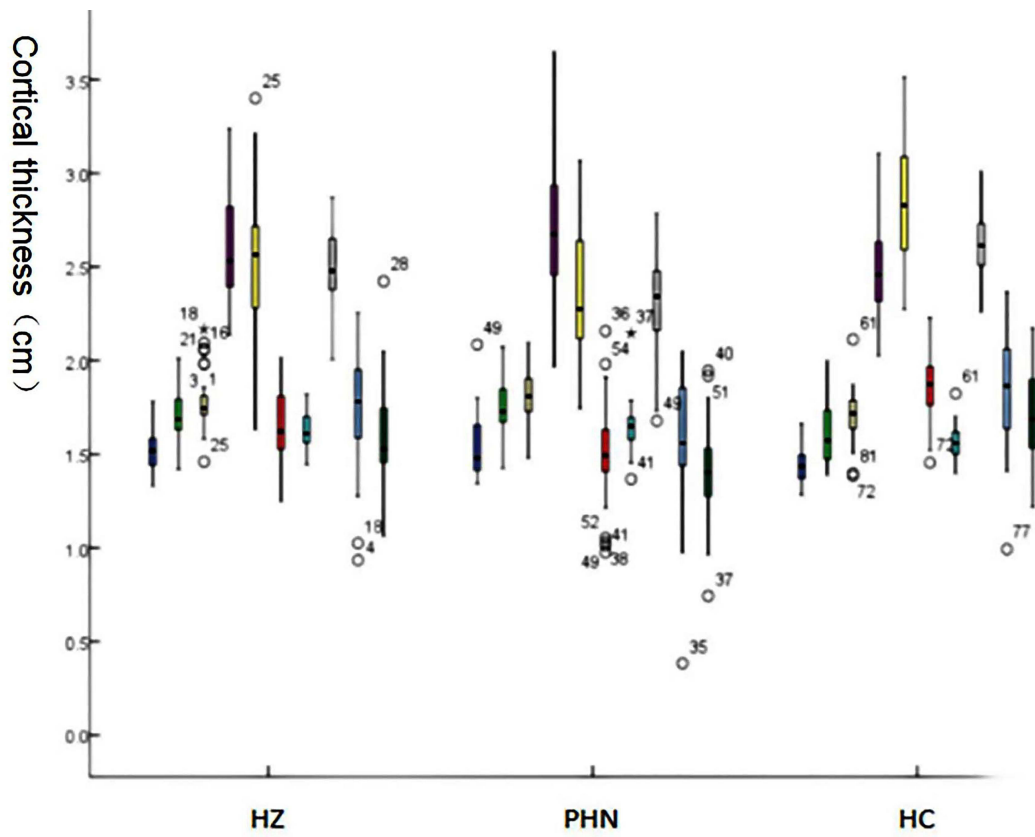

Figure 2 Significant differences in cortical thickness between PHN patients $(n=30)$ and HCs $(n=30)$.

Notes: The y-coordinate represents cortical thickness $(\mathrm{cm})$. Average cortical thickness and degree of variation: the horizontal line in the middle of the figure represents the median; the upper and lower line segments represent the third quartile and the first quartile, respectively; and the top and bottom asterisks and circles represent extreme values.

Abbreviations: HZ, herpes zoster; PHN, postherpetic neuralgia; HC, healthy control; LVI, LV2, left primary visual cortex; L3A, L3B, left somatosensory and motor cortex; LFOP4, left insular and frontal opercular cortex; RVI, RV2, RV3, right primary visual cortex; RS32, right anterior cingulate and medial prefrontal cortex; RSTV, right superior temporal visual cortex. 


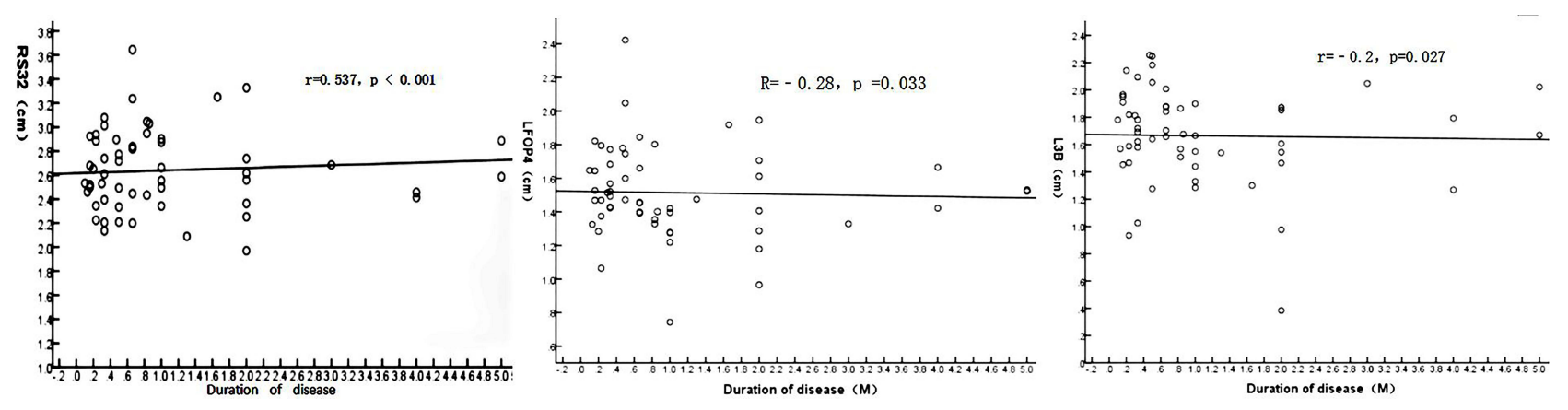

Figure 3 Pearson correlation between the duration of disease $(M)$ and cortical thickness $(\mathrm{cm})$ of RS32 $(r=0.537, P<0.00 \mathrm{I})$, LFOP4 $(r=-0.28, P=0.033)$, and L3B $(r=-$ $0.2, \mathrm{P}=0.027)$ in $\mathrm{HZ}$ and $\mathrm{PHN}$ patients.

Abbreviations: M, month; RS32, right anterior cingulate and medial prefrontal cortex; LFOP4, left insular and frontal opercular cortex; L3b, left motor cortex.

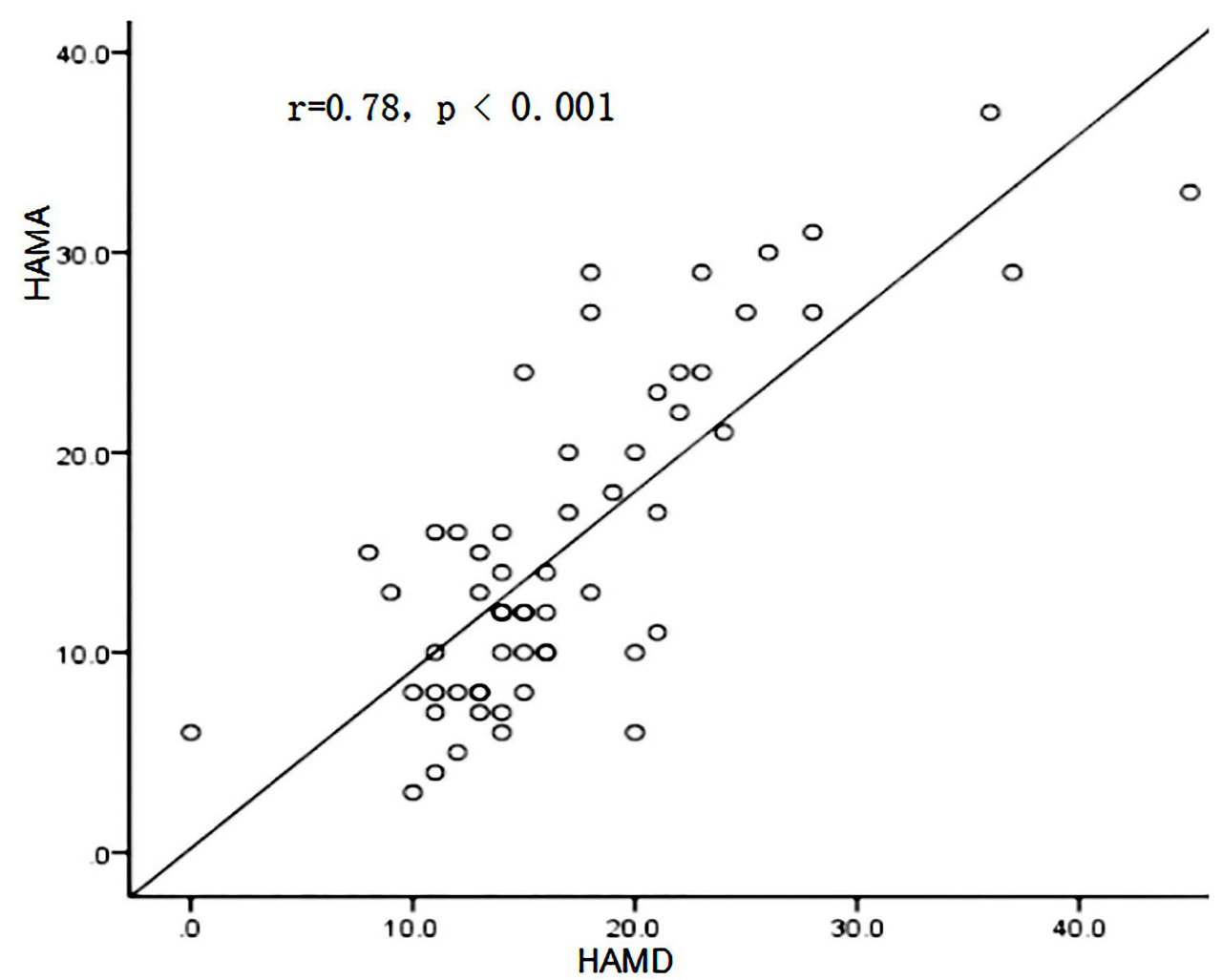

Figure 4 Pearson correlation between HAMA scores (ordinate) and HAMD scores (abscissa) in $\mathrm{HZ}$ patients and $\mathrm{PHN}$ patients $(r=0.78, \mathrm{P}<0.00 \mathrm{I}$ ).

Abbreviations: HAMA, Hamilton Anxiety Scale; HAMD, Hamilton Depression Scale; HZ, herpes zoster; PHN, postherpetic neuralgia.

patients, which was consistent with the results of previous $\mathrm{DKI}^{5}$ and $\mathrm{VBM}^{6,7}$ studies. Studies have shown that PHN, somatoform pain disorder, trigeminal neuralgia, chronic pain disorder and chronic low back pain are types of chronic neuropathic pain. ${ }^{22}$ It was found that the thickness of the insular and temporal cortex decreased in different types of chronic pain patients, indicating that changes in insular and temporal cortex thickness may be the common mechanism of pain in different types of pain-related diseases. This study also found that there was a negative correlation between the cortical thickness of LFOP4 and the duration of disease in patients with HZ and PHN, indicating that the decrease in cortical thickness of LFOP4 may be related to the progression of pain, and the cortical thickness of LFOP4 may be an important indicator of the conversion from HZ to PHN. In addition, studies have shown that changes in insular cortical thickness are related to depression, anxiety disorders, bipolar disorder, subjective cognitive impairment, clinical symptoms and neurocognitive deficits in psychiatric patients. ${ }^{23,24}$ The temporal lobe is also considered to play a very 
important role in mood regulation disorders (anxiety and depression). ${ }^{18,19}$ It can be inferred that the anxiety and depression symptoms of PHN patients may be related to decreases in the thickness of LFOP4 and RSTV.

The primary visual cortex was previously considered to be solely a visual processing region. However, the latest research shows that the human primary visual cortex is a site of multisensory processing, ${ }^{25}$ and the visual cortex participates in many visuomotor integration processes. ${ }^{26}$ Previous magnetic resonance studies on the visual cortex have found that patients with schizophrenia and bipolar disorder have abnormal cortical thickness in the visual network, which is related to visual perception defects. ${ }^{27}$ An increase in cortical thickness in the V2 and V3 areas may be related to visual aura in female migraine patients, ${ }^{28}$ and chronic pain such as trigeminal neuralgia may cause thickening of the primary visual cortex. ${ }^{17}$ It can be inferred that the increased thickness of the bilateral primary visual cortex (V1 and V2) in PHN patients may be related to the abnormal visual perception caused by chronic pain.

The RS32 is located in the prefrontal cortex and forms part of the default mode network (DMN). This region participates in various forms of sensory stimulation processing and plays an important role in emotion, cognitive processing and pain response. ${ }^{29,30}$ Previous studies have shown that changes in gray matter volume, cortical thickness and neural network activity in the prefrontal lobe and cingulate gyrus of patients with chronic neuropathic pain diseases such as chronic back pain, ${ }^{31}$ chronic headache $(\mathrm{CH}),{ }^{32}$ and trigeminal neuralgia $(\mathrm{TN})^{21}$ are related to emotion and pain management caused by chronic pain. ${ }^{33}$ In the present study, the cortical thickness of RS32 increased in PHN patients, indicating that the change in RS32 cortical thickness in PHN patients is related to anxiety and depression. This study found that in HZ and PHN patients, HAMA and HAMD scores had no direct correlation with the duration of disease, while the increase in RS32 cortical thickness, which was also responsible for anxiety and depression, was correlated with the duration of disease, indicating that the changes in cortical thickness in brain areas related to negative emotional states such as anxiety and depression were affected by the course of disease.

Although previous $\mathrm{DKI}^{5}$ and $\mathrm{VBM}^{6,7}$ studies on $\mathrm{HZ}$ and $\mathrm{PHN}$ patients did not find structural changes in the primary visual cortex, anterior cingulate cortex or medial prefrontal cortex, the present study found increased cortical thickness in these regions. Studies have shown that there are two categories of possible reasons for a change in cortical thickness. One category consists of pathological reasons, such as repeated painful stimulation, which leads to an increase in neuronal excitability and glial cell activation in some brain regions, resulting in an increase in cortical thickness. ${ }^{34}$ The second category consists of physiological reasons, that is, the change in cortical thickness is affected by physiological factors such as cerebral perfusion. ${ }^{35}$ Since previous studies on patients with chronic neuropathic pain ${ }^{17,21,28,31,32}$ have shown that the increase in the thickness of the primary visual cortex, anterior cingulate cortex and medial prefrontal cortex in patients with PHN is a pathological change, it is reasonable to speculate that the increase in the thickness of the bilateral primary visual cortex, right anterior cingulate cortex and medial prefrontal cortex in patients with PHN is due to chronic pain, anxiety, depression and other pathological emotional states.

The somatosensory and motor cortex play a significant role in the perception, localization and regulation of pain. ${ }^{36}$ Studies have shown that patients with trigeminal neuropathic pain ${ }^{17}$ and patients with chronic low back pain ${ }^{37}$ have increased activation and thickness of the primary somatosensory cortex during nociceptive stimuli such as normal and pathological pain. The increase in the cortical thickness of the L3A in PHN patients indicates that long-term chronic pathological pain in PHN patients leads to activation of the primary somatosensory cortex, which may be the cause of hypersensitivity to pain in this population. In patients with trigeminal neuropathic pain, the primary motor cortex contralateral to the pain becomes thinner, indicating that pain can inhibit the motor cortex. ${ }^{38,39}$ The decrease in L3B cortical thickness in PHN patients indicates that PHN patients may avoid neuropathic pain by inhibiting motor function. A VBM study of $\mathrm{HZ}$ and PHN found that the GMV of the somatic and motor cortex was decreased in PHN patients, ${ }^{7}$ which is inconsistent with the results of this study. There may be many reasons for the inconsistency of these cortical morphometry results; the main reason for the different results may be different sample characteristics or research methods. ${ }^{40}$ Studies have shown that the accuracy of VBM in spatial positioning is only $35 \%$ as high as that of $\mathrm{SBM} ;{ }^{40}$ that is, the SBM can achieve more accurate spatial positioning than traditional VBM, yielding more reliable results, but further research is needed.

ANOVA (uncorrected) between groups found that the cortical thickness of LFOP4 and L3B tended to be lower in PHN patients than in HZ patients, and the cortical thickness of LFOP4 and L3B was negatively correlated with the duration of disease in HZ and PHN patients (Figure 3), that is, the longer the duration of pain in HZ and PHN 
patients, the thinner the cortex in these regions, indicating that cortical thinning in LFOP4 and L3B may be a key indicator of the conversion from HZ to PHN. Compared with HCs, HZ patients showed increased cortical thickness in V1, V2, and L3A in HZ patients, while the cortical thickness of RSTV and L3B decreased. It may be that the duration of pain in $\mathrm{HZ}$ patients is short $(0.35 \pm 0.17$ months), preventing the cortical thickness from changing significantly.

\section{Limitations}

There are several limitations of the study. First, we used a cross-sectional study design. Although changes in cortical morphological index thickness in HZ and PHN patients were detected, we could not clarify the causal relationship between the changes in cortical thickness and the persistence of $\mathrm{HZ}$ pain. We could not determine whether pain caused changes in cerebral cortical thickness in $\mathrm{HZ}$ and PHN patients or whether changes in cortical thickness facilitated progression from HZ to PHN. In addition, SBM is not suitable for examining the deep gray matter nuclei of the brain, including the thalamus, basal ganglia and amygdala. Moreover, no study has separately analyzed patients with different types and sites of pain. In the future, an expanded sample size should be used to study patients with different

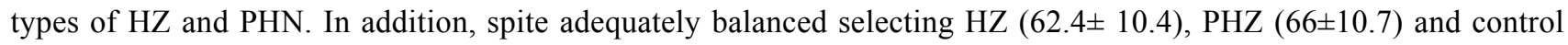
group (63 \pm 9.0 ), and age was used as covariate in ANOVA between groups, the effect of age on cortical thickness should still be considered. In the future, we will try to select matched HZ and PHN patients and control group with a small age range.

\section{Conclusion}

In conclusion, this study used SBM to study and analyze the cortical thickness of patients with HZ and PHN. We found that the cortical thickness of PHN patients was altered in the areas related to sensory, motor, and cognitive/emotional changes, which affected the process of brain neuroplasticity. These alterations may explain the transformation of $\mathrm{HZ}$ into PHN and provide a neuropathological mechanism for the persistence of pain in PHN patients.

\section{Acknowledgments}

This study was supported by grants from the National Natural Science Foundation of China (Grant No. 81960313), the Nature Science Foundation of Jiangxi Province (grant no. 20181BAB205028 and grant no. 20192BAB205039).

\section{Author Contributions}

All authors made a significant contribution to the work reported, whether that is in the conception, study design, execution, acquisition of data, analysis and interpretation, or in all these areas; took part in drafting, revising or critically reviewing the article; gave final approval of the version to be published; have agreed on the journal to which the article has been submitted; and agree to be accountable for all aspects of the work.

\section{Disclosure}

The authors report no conflicts of interest in this work.

\section{References}

1. Gershon AA, Breuer J, Cohen JI, et al. Varicella zoster virus infection. Nat Rev Dis Primers. 2015;1:15016. doi:10.1038/nrdp.2015.16

2. Baron R, Binder A, Wasner G. Neuropathic pain: diagnosis, pathophysiological mechanisms, and treatment. Lancet Neurol. $2010 ; 9: 807-819$. doi:10.1016/S1474-4422(10)70143-5

3. Chen F, Chen F, Shang Z, et al. White matter microstructure degenerates in patients with postherpetic neuralgia. Neurosci Lett. 2017;656:152-157. doi:10.1016/j.neulet.2017.07.023

4. Dai H, Jiang C, Wu G, et al. A combined DTI and resting state functional MRI study in patients with postherpetic neuralgia. Jpn J Radiol. 2020;38:440-450. doi:10.1007/s11604-020-00926-4

5. Zhang Y, Yu T, Qin B, et al. Microstructural abnormalities in gray matter of patients with postherpetic neuralgia: a diffusional kurtosis imaging study. Pain Physician. 2016;19:E601-E611.

6. Wang SD, Lu Y, Zhao W, et al. Observation of morphological changes of brain gray matter volume in patients with postherpetic neuralgia using VBM-DARTEL method. Pract Radiol. 2017;33(9):1337-1340.

7. Liu J, Gu L, Huang Q, et al. Altered gray matter volume in patients with herpes zoster and postherpetic neuralgia. J Pain Res. 2019;12:605-616. doi:10.2147/JPR.S183561 
8. Lerch JP, van der Kouwe André JW, Raznahan A, et al. Studying neuroanatomy using MRI. Nat Neurosci. 2017;20:314-326. doi:10.1038/nn.4501

9. Browning M, Fletcher P, Sharpe M. Can neuroimaging help us to understand and classify somatoform disorders? A systematic and critical review. Psychosom Med. 2011;73:173-184. doi:10.1097/PSY.0b013e31820824f6

10. Chiarello C, Vazquez D, Felton A, et al. Structural asymmetry of the human cerebral cortex: regional and between-subject variability of surface area, cortical thickness, and local gyrification. Neuropsychologia. 2016;93:365-379. doi:10.1016/j.neuropsychologia.2016.01.012

11. Tamnes CK, Herting MM, Goddings A-L, et al. Development of the cerebral cortex across adolescence: a multisample study of inter-related longitudinal changes in cortical volume, surface area, and thickness. $J$ Neurosci. 2017;37:3402-3412. doi:10.1523/JNEUROSCI.3302-16.2017

12. Wannan CMJ, Cropley VL, Chakravarty MM, et al. Evidence for network-based cortical thickness reductions in schizophrenia. Am $J$ Psychiatry. 2019;176:552-563. doi:10.1176/appi.ajp.2019.18040380

13. Glasser MF, Smith SM, Marcus DS, et al. The human connectome project's neuroimaging approach. Nat Neurosci. 2016;19:1175-1187. doi: $10.1038 / \mathrm{nn} .4361$

14. Hutton C, Draganski B, Ashburner J, et al. A comparison between voxel-based cortical thickness and voxel-based morphometry in normal aging. Neuroimage. 2009;48:371-380. doi:10.1016/j.neuroimage.2009.06.043

15. Uddin LQ, Nomi JS, Hébert-Seropian B, et al. Structure and function of the human insula. J Clin Neurophysiol. 2017;34:300-306. doi:10.1097/ WNP.0000000000000377

16. Meyer E, Morawa E, Nacak Y, et al. Insular cortical thickness in patients with somatoform pain disorder: are there associations with symptom severity and childhood trauma? Front Psychiatry. 2020;11:497100. doi:10.3389/fpsyt.2020.497100

17. Desouza DD, Moayedi M, Chen DQ, et al. Sensorimotor and pain modulation brain abnormalities in trigeminal neuralgia: a paroxysmal, sensory-triggered neuropathic pain. PLoS One. 2013;8:e66340. doi:10.1371/journal.pone.0066340

18. Chen Y, Shimotake A, Matsumoto R, et al. The 'when' and 'where' of semantic coding in the anterior temporal lobe: temporal representational similarity analysis of electrocorticogram data. Cortex. 2016;79:1-13. doi:10.1016/j.cortex.2016.02.015

19. Magon S, Sprenger T, Otti A, et al. Cortical thickness alterations in chronic pain disorder: an exploratory MRI study. Psychosom Med. 2018;80:592-598. doi:10.1097/PSY.0000000000000605

20. Lebedeva A, Sundström A, Lindgren L, et al. Longitudinal relationships among depressive symptoms, cortisol, and brain atrophy in the neocortex and the hippocampus. Acta Psychiatr Scand. 2018;137:491-502. doi:10.1111/acps.12860

21. Albano L, Agosta F, Basaia S, et al. Alterations of brain structural MRI are associated with outcome of surgical treatment in trigeminal neuralgia. Eur J Neurol. 2022;29(1):305-317. doi:10.1111/ene.15105

22. Gossrau G, Sabatowski R. Diagnostics and therapy of neuropathic pain. Anaesthesist. 2021;70:993-1002. doi:10.1007/s00101-021-01039-x

23. Hatton SN, Lagopoulos J, Hermens DF, et al. Correlating anterior insula gray matter volume changes in young people with clinical and neurocognitive outcomes: an MRI study. BMC Psychiatry. 2012;12:45. doi:10.1186/1471-244X-12-45

24. Youn H, Choi M, Lee S, et al. Decreased cortical thickness and local gyrification in individuals with subjective cognitive impairment. Clin Psychopharmacol Neurosci. 2021;19:640-652. doi:10.9758/cpn.2021.19.4.640

25. Murray MM, Thelen A, Thut G, et al. The multisensory function of the human primary visual cortex. Neuropsychologia. 2016;83:161-169. doi:10.1016/j.neuropsychologia.2015.08.011

26. de Schotten MT, Urbanski M, Valabregue R, et al. Subdivision of the occipital lobes: an anatomical and functional MRI connectivity study. Cortex. 2014;56:121-137. doi:10.1016/j.cortex.2012.12.007

27. Reavis EA, Lee J, Wynn JK, et al. Cortical thickness of functionally defined visual areas in schizophrenia and bipolar disorder. Cereb Cortex. 2017;27:2984-2993. doi:10.1093/cercor/bhw151

28. Gaist D, Hougaard A, Garde E, et al. Migraine with visual aura associated with thicker visual cortex. Brain. 2018;141:776-785. doi:10.1093/brain/ awx382

29. May A. Structural brain imaging: a window into chronic pain. Neuroscientist. 2011;17:209-220. doi:10.1177/1073858410396220

30. Fierro B, De Tommaso M, Giglia F, et al. Repetitive transcranial magnetic stimulation (rTMS) of the dorsolateral prefrontal cortex (DLPFC) during capsaicin-induced pain: modulatory effects on motor cortex excitability. Exp Brain Res. 2010;203:31-38. doi:10.1007/s00221-010-2206-6

31. Fritz H-C, McAuley James H, Wittfeld K, et al. Chronic back pain is associated with decreased prefrontal and anterior insular gray matter: results from a population-based cohort study. J Pain. 2016;17:111-118. doi:10.1016/j.jpain.2015.10.003

32. Yang Q, Wang Z, Yang L, et al. Cortical thickness and functional connectivity abnormality in chronic headache and low back pain patients. Hum Brain Mapp. 2017;38:1815-1832. doi:10.1002/hbm.23484

33. Palermo S, Benedetti F, Costa T, et al. Pain anticipation: an activation likelihood estimation meta-analysis of brain imaging studies. Hum Brain Mapp. 2015;36:1648-1661. doi:10.1002/hbm.22727

34. Liu X, Hou Z, Yin Y, et al. Decreased cortical thickness of left premotor cortex as a treatment predictor in major depressive disorder. Brain Imaging Behav. 2021;15:1420-1426. doi:10.1007/s11682-020-00341-3

35. Fierstra J, Maclean DB, Fisher JA, et al. Surgical revascularization reverses cerebral cortical thinning in patients with severe cerebrovascular steno-occlusive disease. Stroke. 2011;42:1631-1637. doi:10.1161/STROKEAHA.110.608521

36. Davis KD, Moayedi M. Central mechanisms of pain revealed through functional and structural MRI. J Neuroimmune Pharmacol. $2013 ; 8: 518-534$. doi:10.1007/s11481-012-9386-8

37. Kong J, Spaeth RB, Wey H-Y, et al. S1 is associated with chronic low back pain: a functional and structural MRI study. Mol Pain. 2013;9:43. doi:10.1186/1744-8069-9-43

38. DaSilva Alexandre F, Becerra L, Pendse G, et al. Colocalized structural and functional changes in the cortex of patients with trigeminal neuropathic pain. PLoS One. 2008;3:e3396. doi:10.1371/journal.pone.0003396

39. Romaniello A, Cruccu G, McMillan AS, et al. Effect of experimental pain from trigeminal muscle and skin on motor cortex excitability in humans. Brain Res. 2000;882:120-127. doi:10.1016/S0006-8993(00)02856-0

40. Coalson TS, Van Essen David C, Glasser MF. The impact of traditional neuroimaging methods on the spatial localization of cortical areas. Proc Natl Acad Sci U S A. 2018;115:E6356-E6365. doi:10.1073/pnas.1801582115 


\section{Publish your work in this journal}

The Journal of Pain Research is an international, peer reviewed, open access, online journal that welcomes laboratory and clinical findings in the fields of pain research and the prevention and management of pain. Original research, reviews, symposium reports, hypothesis formation and commentaries are all considered for publication. The manuscript management system is completely online and includes a very quick and fair peer-review system, which is all easy to use. Visit http://www.dovepress.com/testimonials.php to read real quotes from published authors.

Submit your manuscript here: https://www.dovepress.com/journal-of-pain-research-journal 